\title{
II mio ricordo del Professor Quirino Maggiore
}

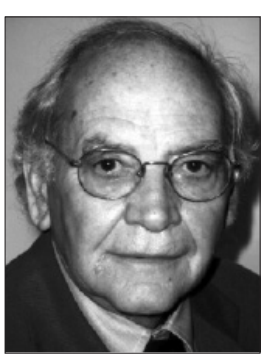

Quirino Maggiore

Con molto rammarico e sincero dolore prendo atto che oggi non è più tra noi un (il mio) maestro, un maestro per tutti quanti con lui hanno collaborato, e non soltanto nella vita professionale.

Per un ricordo compiuto di questa persona non basterebbe un intero numero del Giornale, pertanto proverò a ricordarlo con degli affreschi, solo alcuni, quelli che in questo triste momento ricordo più vividamente, con tenerezza e amore, perché per lui non si poteva non provarne. In realtà, poiché siamo tutti esseri umani (e quindi per definizione imperfetti), talora ci ha fatto provare anche sentimenti contrastanti, ma non c'è dubbio che il bilancio sia stato assolutamente positivo, eccome se lo è stato!

Ricordo che nel 1987, quando arrivò la notizia che il "mitico" Professor Quirino Maggiore sarebbe venuto a Firenze nell'Ospedale Santa Maria Annunziata, capii immediatamente che quella sarebbe stata l'occasione professionale della mia vita e che prima o poi, anche come barelliere, avrei dovuto cercare di prenderla al volo. Partecipato al concorso ed entrato in graduatoria, l'anno successivo il Prof. mi chiamò per conoscermi prima di prendere servizio. Già al primo impatto mi resi conto di trovarmi dinanzi a un uomo dal grande carisma, e con una "testa" diversa da tanti altri. Questo ultimo aspetto divenne subito chiaro perché mi chiese "solo" di comprare e studiare due volumi. Ma non si trattava di testi di nefrologia o di medicina interna, come mi sarei aspettato. I due volumi richiesti erano uno di epidemiologia (1) e l'altro di statistica (2). Poi con aria sorniona e distratta (un'arte che ben ricorderà chi lo ha conosciuto) mi disse, con la sua fedele pipa in mano, "... e mi raccomando, Lombardi, fai anche un corso di bella calligrafia" a sancire che quell'uomo dallo sguardo profondo e intelligente, o la sapeva lunga su di me tanto da sapere che scrivevo (e scrivo) con una gallino-grafia (si era informato dunque), o era capace di cogliere in chi gli stava davanti cose che altri non riuscivano a percepire.

Era un personaggio integerrimo, leale e onesto, austero, financo aggressivo verso chi si limitava a un'attività di basso profilo nella professione o anche nella vita di tutti i giorni. Prediligeva l'impegno sociale anche per i suoi allievi e l'impegno professionale doveva prevaricare se necessario ogni altra attività, un "devo perché devo" di kantiana memoria. L'orario di lavoro era un concetto che per lui non esisteva (e quindi neanche per noi che accumulavamo migliaia di ore in più... io sto cercando di smaltirne ancor oggi!). In questo contesto anche l'espressone "tengo famiglia" era tabù e non compatibile con lo svolgimento dell'attività professionale. In ospedale dovevamo arrivare "già mangiati, pisciati e cacati" per non perder tempo in funzioni corporali di bassa utilità al reparto!

Ma in mezzo a complicati ragionamenti fisio-patologici era anche capace di battute spiritose e sagaci, che a un giovincello come me sembravano ottocentesche ma che crescendo (invecchiando) mi sono trovato a cercare spesso di emulare. Severo, sì, ma giusto. Severo anche con se stesso, anzi direi molto di più con se stesso che con gli altri. La sua severità sembrava direttamente proporzionale alla stima che nutriva per i suoi collaboratori. Questi (noi) sapevano bene che questa poteva sfiorare la drammaticità per errori di leggerezza o pressappocaggine in corsia, nella ricerca o anche solo nel redigere anamnesi ("Ragazzi ricordatevi che la diagnosi la farete al 70\% grazie a un'anamnesi fatta bene").

La sua severità era esemplare nella correzione dei manoscritti, anche se avessimo dovuto pubblicare sul Corriere dei Piccoli. Ricordo il primo lavoro pubblicato sotto la sua guida (3) sul primo numero del Journal of Nephrology di cui il Prof., come sappiamo tutti, è stato fondatore e primo Guest Editor insieme a personaggi del calibro di Giuseppe Andres, Netar Mallick, Giuseppe Maschio, Giuseppe Remuzzi ed Eberhard Ritz, tanto per citarne alcuni.

Non so dire quante revisioni di quel manoscritto abbia dovuto fare (peccato non averle conservate), tante, tantissime, tanto che alla fine il lavoro era oramai praticamente scritto tutto di suo pugno. Quando andavo con i fogli dattiloscritti in mano nella sua stanza, sicuro di aver raggiunto la versione definitiva, c'era sempre qualche sorpresa. Correzioni su correzioni, con quella calligrafia così minuta e capace di andare in ogni direzione, con curve e inclinazioni impossibili, correzioni scritte con una delle sue penne stilo o comunque a inchiostro, nuove correzioni e migliorie che fiorivano continuamente come margherite a primavera! E chi aveva poi il coraggio di tornare nella sua stanza a disturbarlo, per chiedergli "Prof. ma cosa c'è scritto qui?". Così si veniva via dalla sua stanza al profumo di tabacco di pipa (che lui manovrava con grande destrezza, con quelle mani magre e forti), sconsolati e abbattuti perché il Prof. non era contento neppure lui di quel che alla fin fine aveva scritto: riusciva a correggersi e migliorarsi continuamente, in una specie di moto perpetuo che non lasciava mai intravedere una fine. Si usciva in silenzio dalla 
sua stanza e dalla nuvola di fumo, pensando "Va a finire che lo pubblica prima qualcun altro!".

In quei momenti mi sembrava di vedere Dante Alighieri in camice bianco, curvo sui fogli del manoscritto e pensavo: doveva aver seguito anche lui il metodo del Prof. per scrivere cotanta roba!

Alla fin fine c'era comunque sempre un insegnamento nel suo modo di fare, anzi di essere, che non era ricercato o voluto, bensi spontaneo e naturale. Nelle innumerevoli correzioni di ogni paragrafo era insito un concetto ben preciso: mai accontentarsi perché cercare sempre di fare meglio è possibile, è normale, è dovuto, è morale!

Ricordo un altro paio di aneddoti, e con questi cerco di concludere perché potrei/dovrei scrivere ancora molto. Una sera, a una cena di reparto, mentre il Prof. stava parcheggiando la sua BMW serie 5, rigorosamente di seconda se non terza mano (e che aveva sostituito la precedente Volvo station-wagon a gas, tanto per intenderci), mi ero improvvisato scherzosamente parcheggiatore: "Venga Prof., ancora Prof., basta così Prof. ... no basta Prof., basta, c'è l'alberooo!!!". Bum!!! Uscendo dalla macchina, con quel suo modo di fare che non sapevi mai se dovevi temere o metterti a ridere, mi gridò "Lombardi, resterai sempre un assistente!". E quando, un giorno mentre andavamo tutti a mensa, trovai il coraggio di chiedergli "Prof. sono stato a Genova a vedere la scuola di specializzazione in diabetologia, vorrei iscrivermici... che ne pensa?", mi rispose "Marco, ma se devi ancora imparare a fare il nefrologo!... Fai una cosa per volta!".

Caro Prof., avevi proprio ragione. Non credo che mi iscriverò mai a un'altra scuola di specializzazione. E non solo per l'età, ma perché nonostante i tuoi insegnamenti devo davvero ancora imparare a fare il nefrologo... almeno secondo i canoni che tu riconoscevi come dovuti!

Non ti dimenticherò Prof., mai.

Marco Lombardi Editor in Chief

Giornale di Tecniche Nefrologiche e Dialitiche

Firenze, 2 settembre 2017

\section{Bibliografia essenziale}

1. Sackett DL, Haynes RB, Tugwell P, Guyatt G. Clinical epidemiology. A basic science for clinical medicine. Boston, MA: Little Brown \& Co 1985.

2. Bland M. An introduction to medical statistics. Oxford: Oxford Mediacl Publications 1987.

3. Salvadori M, et al. Acute renal involvement in Hantavirus infections: First report in Italy. J Nephrol. 1989;2(1):17-22.

\section{Letture e video consigliati}

Timio M. Intervista al prof. Quirino Maggiore: un nefrologo "nomen omen". G Ital Nefrol. 2012;29(4):484-7.

Fogazzi GB. Intervista a Quirino Maggiore. In: Interviste con la storia della Nefrologia Italiana di Schena FP e Fogazzi GB. Milano: Wichtig Publishing, 2016:108-15.

http://web.era-edta.org/quirino-maggiore 